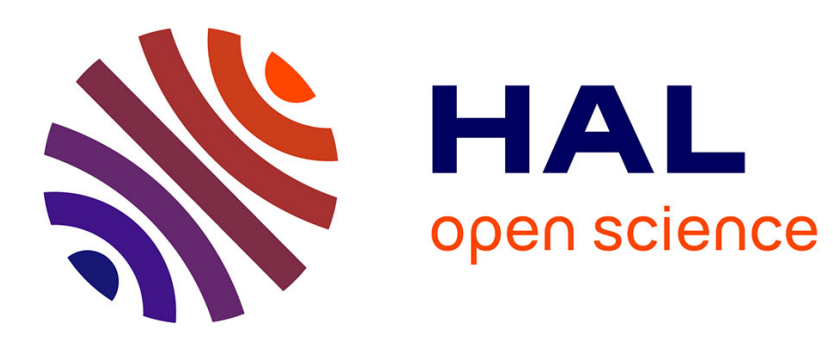

\title{
Recherches sur les causes de l'exclusion du sodium des feuilles des plantes sensibles à $\mathrm{NaCl}$
}

Frej Slama

\section{To cite this version:}

Frej Slama. Recherches sur les causes de l'exclusion du sodium des feuilles des plantes sensibles à $\mathrm{NaCl}$. Agronomie, 1987, 7 (7), pp.517-522. hal-00885021

\section{HAL Id: hal-00885021 \\ https://hal.science/hal-00885021}

Submitted on 1 Jan 1987

HAL is a multi-disciplinary open access archive for the deposit and dissemination of scientific research documents, whether they are published or not. The documents may come from teaching and research institutions in France or abroad, or from public or private research centers.
L'archive ouverte pluridisciplinaire HAL, est destinée au dépôt et à la diffusion de documents scientifiques de niveau recherche, publiés ou non, émanant des établissements d'enseignement et de recherche français ou étrangers, des laboratoires publics ou privés. 


\title{
Recherches sur les causes de l'exclusion du sodium des feuilles des plantes sensibles à $\mathrm{NaCl}$
}

\author{
Frej SLAMA \\ Institut National Agronomique, Laboratoire d'Agronomie, 43, avenue Charles-Nicolle, 1002 Tunis, Tunisie
}

RÉSUMÉ

\begin{abstract}
La comparaison des distributions de $\mathrm{Na}^{+}$dans 10 plantes glycophytes cultivées sur milieu liquide, montre que l'exclusion de $\mathrm{Na}^{+}$des feuilles est d'autant plus prononcée que les plantes sont plus sensibles à $\mathrm{NaCl}$. Les feuilles détachées des plantes les plus sensibles absorbent en $24 \mathrm{~h}$ à travers les pétioles moins de $\mathrm{Na}^{+}$que celles des plantes tolérantes. Néanmoins, les quantités ainsi absorbées sont très supérieures à celles que les feuilles en place accumulent en plusieurs jours de culture en présence de $\mathrm{NaCl}$. Chez les plantes tolérantes, cette différence entre les feuilles détachées et en place ne s'observe pas. Le $\mathrm{Na}^{+}$absorbé par les feuilles détachées reste préférentiellement associé aux nervures chez les plantes sensibles, et aux limbes chez les plantes tolérantes. Enfin, la mesure de la vitesse d'incorporation de ${ }^{24} \mathrm{Na}^{+}$par des tranches de limbes de haricot (très sensible), et de cotonnier (tolérant), montre que l'influx de $\mathrm{Na}^{+}$dans les cellules de mésophylle est paradoxalement plus élevé chez la plante sensible que chez la plante tolérante. La conclusion est que l'exclusion de $\mathrm{Na}^{+}$, caractéristique des feuilles en place des plantes sensibles, est atténuée chez les feuilles détachées, ęt disparaît au niveau des limbes isolés. La rétention et la redistribution de $\mathrm{Na}^{+}$par les systèmes vasculaires des tiges, pétioles et nervures semblent être des facteurs importants de la protection des feuilles des plantes sensibles contre l'accumulation de cet ion.
\end{abstract}

Mots clés additionnels : Transports, Cucumis, Cucurbita, Gossypium, Helianthus, Phaseolus.

Comparison of $\mathrm{Na}^{+}$distribution in ten glycophytes cultivated hydroponically showed that $\mathrm{Na}^{+}$exclusion from leaves was positively correlated with sensitivity to $\mathrm{NaCl}$. When excised, the leaves of $\mathrm{NaCl}$-sensitive plants absorbed in $24 \mathrm{~h}$ through their petioles smaller amounts of $\mathrm{Na}^{+}$than did the leaves of $\mathrm{NaCl}$-tolerant plants. Nevertheless, these amounts were larger than those present in the leaves of plants cultivated for several days in the presence of $\mathrm{NaCl}$. This difference of $\mathrm{Na}^{+}$accumulations in excised leaves and in leaves in situ was not observed with $\mathrm{NaCl}$-tolerant plants. After absorption by excised leaves, $\mathrm{Na}^{+}$was preferentially accumulated in the veins in $\mathrm{NaCl}$-sensitive plants, and in the lamina in $\mathrm{NaCl}$-tolerant ones. $\mathrm{The}^{24} \mathrm{Na}^{+}$influx into lamina slices was higher in $\mathrm{NaCl}$-sensitive plants than in the others. It was concluded that $\mathrm{Na}$ ' exclusion, which is characteristic of attached leaves of $\mathrm{NaCl}$-sensitive plants, is attenuated in excised leaves, and disappears when isolated laminas are used. The retention and the recirculation of $\mathrm{Na}^{+}$by the vascular tissues of stems, petioles and leaf veins are probably important for protecting the leaves of $\mathrm{NaCl}$-sensitive plants against $\mathrm{Na}^{+}$ accumulation.

Additional key words : Transport, Cucumis, Cucurbita, Gossypium, Helianthus, Phaseolus.

\section{INTRODUCTION}

Les glycophytes les plus sensibles à $\mathrm{NaCl}$ n'accumulent que très peu de $\mathrm{Na}^{+}$dans leurs feuilles (COLLANDER, 1941 ; Bernstein, 1964). Par exemple, chez le haricot, très sensible, l'accumulation de $\mathrm{Na}^{+}$décroît selon la séquence racines-tiges-feuilles (WALLACE, 1963 ; HAMZA, 1977 ; Slama, 1982). Au contraire, les glycophytes tolérants et les halophytes transportent aisément $\mathrm{Na}^{+}$dans leurs feuilles. Par exemple, chez le cotonnier, glycophyte tolérant cultivé en présence de
$\mathrm{NaCl}$, les feuilles sont plus riches en $\mathrm{Na}^{+}$que les tiges et les racines (PEARSON, 1964 ; Slama, 1974). On désigne par «exclusives" (excluder) les plantes qui n'accumulent pas $\mathrm{Na}^{+}$dans les mésophylles, et par " inclusives » (includer) les autres (YEO, 1983).

A priori, la protection des feuilles contre l'envahissement par $\mathrm{Na}^{+}$peut être assurée par un contrôle de l'absorption nette par la racine. Nous avons procédé à une étude de cette question sur 12 plantes différant par leur sensibilité à $\mathrm{NaCl}$, inclusives et exclusives (Slama, 1986). Les racines des 2 types de plantes 
absorbent $\mathrm{Na}^{+}$à des vitesses comparables, mais le conduisent différemment dans le xylème. Le cyanure ou le froid, appliqués sur les racines, ralentissent le transfert de $\mathrm{Na}^{+}$vers les parties aériennes chez les inclusives, mais l'accélèrent chez les exclusives. La conclusion générale est que les racines des plantes sensibles sont moins efficaces pour introduire $\mathrm{Na}^{+}$dans le xylème, et plus efficaces pour le retenir dans leurs tissus que celles des plantes tolérantes. Néanmoins, ces propriétés ne peuvent expliquer les différences d'accumulation foliaire de $\mathrm{Na}^{+}$observées sur de longues durées de culture, en fonction du degré de sensibilité à $\mathrm{NaCl}$. Nous présentons ici une étude destinée à définir les responsabilités respectives des feuilles et des autres organes dans ce phénomène. Cette étude porte sur dix plantes étudiées précédemment (SlAMA, 1986).

\section{MATÉRIELS ET MÉTHODES}

\section{A. Matériels}

L'étude porte sur 10 plantes cultivées, correspondant à 6 espèces ou genres, choisies pour leurs sensibilités variées à la salinité $(\mathrm{NaCl})$. Dans chaque espèce ou genre, 2 cultivars ont été retenus, choisis sur la base de leurs sensibilités différentes à l'irrigation par l'eau salée $(\mathrm{NaCl})$ au champ. La liste de ces plantes, et leurs symboles à 2 lettres utilisés ci-après sont donnés dans le tableau 1. L'échelle de sensibilité a été établie en comparant les effets de $\mathrm{NaCl}\left(3 \mathrm{~g} . \mathrm{I}^{-1}\right)$ sur la production de matière sèche en $20 \mathrm{j}$ de culture (SlamA, 1986). De la plus tolérante à la plus sensible, les plantes se classent ainsi : $\mathrm{Ga}, \mathrm{Mc}, \mathrm{Tr}, \mathrm{Hl}, \mathrm{Ti}, \mathrm{G} 5, \mathrm{Mq}$, $\mathrm{Cb}, \mathrm{Hg}, \mathrm{Cl}$. Les expériences d'incorporation de ${ }^{24} \mathrm{Na}^{+}$ ont été faites sur le haricot variété Menil (Tézier, France), analogue à $\mathrm{Hg}$ en ce qui concerne la sensibilité à $\mathrm{NaCl}$, et sur le cotonnier à fibres courtes variété BJA 592 (IRCT), analogue à Ga.

\section{B. Conditions de culture}

Les semis sont faits sur du sable lavé par $\mathrm{HCl}$ et rincé à l'eau déminéralisée. Lorsque l'axe hypocotylé est dressé, les plantules sont transférées sur milieu liquide dans des pots en matière plastique (solution standard dite $S$ ). Le milieu de culture est aéré au moyen de pompes d'aquarium, et renouvelé tous les 3 ou $4 \mathrm{j}$. La composition de la solution $\mathrm{S}$ est la suivante $(\mathrm{mN}): \mathrm{NH}_{4}^{+} 2 ; \mathrm{K}^{+} 5,5 ; \mathrm{Ca}^{2+} 8,1 ; \mathrm{Mg}^{2+} 4,4 ;$ $\mathrm{NO}_{3}^{-} 12 ; \mathrm{SO}_{4}^{2-} 5,7 ; \mathrm{H}_{2} \mathrm{PO}_{4}^{2-} 0,5$. La solution contient également du fer (JACOBSON, 1951) et des oligoéléments. La solution $\mathrm{S} 3$ contient en plus $\mathrm{NaCl} 3 \mathrm{~g} .1^{-1}$. Le $\mathrm{pH}$ des deux solutions est 6.2. L'éclairement est assuré par des lampes à incandescence et des tubes fluorescents (rayonnement actif environ $400 \mathrm{~mol} . \mathrm{s}^{-1}$. $\mathrm{m}^{-2}$ ). L'héméropériode est de $12 \mathrm{~h}$, et la température est $28^{\circ} \mathrm{C} \pm 3{ }^{\circ} \mathrm{C}$ le jour et $18^{\circ} \mathrm{C} \pm 3{ }^{\circ} \mathrm{C}$ la nuit. L'humidité relative est de 50 p. $100 \pm 5$ p. 100 le jour, et 70 p. $100 \pm 5$ p. 100 la nuit.

\section{Mesure de la vitesse de l'absorption nette par les feuilles détachées}

Les feuilles primaires ou cotylédonaires de plantes cultivées depuis $6 \mathrm{j}$ sur milieu $\mathrm{S}$ sont prélevées avec leurs pétioles et des fragments de tiges de $2 \mathrm{~cm}$, qui sont plongés dans un milieu S3 aéré. L'expérience commence au début de l'héméropériode. Elle dure $24 \mathrm{~h}$, dans les mêmes conditions de température, éclairage et humidité que les cultures.

\section{Mesure de l'incorporation de ${ }^{24} \mathrm{Na}^{\dagger}$ par les limbes}

Les cinétiques d'influx de ${ }^{24} \mathrm{Na}^{+}$sont déterminées sur les limbes des feuilles primaires (haricot) ou cotylédonaires (cotonnier) prélevées sur des plantes cultivées depuis $8 \mathrm{j}$ sur milieu $\mathrm{S}$. Une expérience préliminaire a montré que les feuilles cotylédonaires du cotonnier ont le même comportement (absorption de $\mathrm{Na}^{+}$) que les feuilles primaires ; ces dernières n'ont pas été retenues pour l'étude complète car elles sont très petites. Des tranches épaisses de $450 \mu \mathrm{m}$ sont découpées avec un microtome à main dans les régions du limbe sans nervures saillantes et éloignées des bords. Elles sont lavées dans de l'eau distillée et réparties par lots de 100 (10 mg MS environ), dans des sachets de toile de nylon. Les teneurs en $\mathrm{Na}^{+}$pour les deux espèces sont $0,07 \pm 0,02 \mu \mathrm{mol} . \mathrm{g}^{-1} \mathrm{MS}$. Les lots sont prétraités

TABLEAU 1

Liste des plantes utilisées. Le haricot local (Hl) est grimpant, et relativement tolérant à $\mathrm{NaCl}$. La courge locale (Cl), variété Bjewi est sensible au sel. Le melon Qalawi (Mq) est moins robuste que le melon Charentais. Le cotonnier Akala (Ga) est une variété à fibre courte, et le cotonnier 54$76 J$ (G5), une variété à fibres longues.

Plants used in the study. $\mathrm{Hl}$ is a climbing bean, relatively tolerant to $\mathrm{NaCl}$. The pumpkin $\mathrm{Cl}$, variety so-called "Bjewi ", is sensitive to $\mathrm{NaCl}$. The melon $M q$ is less hardy than Mc. The cotton Akala (Ga) is a short-fiber variety, and 54-76 J (G5) is a long-fiber one.

Espèces, cultivars (a), et sensibilité à $\mathrm{NaCl}$

\begin{tabular}{|c|c|c|c|c|c|}
\hline \multirow{2}{*}{\multicolumn{2}{|c|}{ Genres }} & \multicolumn{4}{|c|}{ Espèces, cultivars $\left({ }^{a}\right)$, et sensibilité à $\mathrm{NaCl}$} \\
\hline & & \multicolumn{2}{|r|}{ Plus tolérants } & \multicolumn{2}{|c|}{ Plus sensibles } \\
\hline Haricots & Phaseolus & vulgaris $\mathbf{L}$. & Local $(\mathrm{Hl})$ & vulgaris $\mathrm{L}$. & Gabriella (Hg) \\
\hline Courges & Cucurbita & pepo L. & Courgette blanche $(\mathrm{Cb})$ & maxima $\mathrm{L}$ & Courge Bjewi (Cl) \\
\hline Tournesols & Helianthus & annuus $\mathbf{L}$. & Rekord (Tr) & annuus $\mathrm{L}$. & Issanka (Ti) \\
\hline Melons & Cucumis & melo L. & Charentais (Mc) & melo L. & Qalawi (Mq) \\
\hline Cotonniers & Gossypium & hirsutum $\mathrm{L}$ & Akala (Ga) & barbadense L. & $54-76 \mathrm{~J}(\mathrm{G} 5)$ \\
\hline
\end{tabular}

(a) Les semences proviennent des maisons suivantes: Asgrow, France (Hg) ; Tezier, France (Cb) ; Codisol, France (Tr et Ti) ; INRAT (Mc) ; IRCT, France (Ga et G5). Hl, Cl et MG sont obtenus auprès des cultivateurs Tunisiens. 
pendant 30 min dans une solution aérée de $\mathrm{CaSO}_{4}$ $0,5 \mathrm{mM}$, puis plongés dans $250 \mathrm{ml}$ d'une solution d'absorption aérée à $24{ }^{\circ} \mathrm{C}$. Les solutions d'absorption contiennent $\mathrm{CaSO}_{4} 0,5 \mathrm{mM}$, et $\left({ }^{24} \mathrm{Na}^{+}\right)-\mathrm{NaCl} 0,01 \mathrm{mM}$ à $50 \mathrm{mM}\left(1 \mathrm{mCi} \cdot 1^{-1}\right)$. Après $1 \mathrm{~h}$, les lots sont retirés des solutions radioactives et lavés pendant $30 \mathrm{~min}$ dans $250 \mathrm{ml}$ d'une solution aérée de $\mathrm{CaSO}_{4} 0,5 \mathrm{mM}, \mathrm{NaCl}$ $1 \mathrm{mM}$, à $2^{\circ} \mathrm{C}$. Toutes les opérations se font sous un éclairement de $470 \mu \mathrm{mol} . \mathrm{s}^{-1} \cdot \mathrm{m}^{-2}$ (rayonnement actif). La radioactivité libérée dans le milieu de lavage mesure la fraction réversible de l'absorption. L'influx (vitesse d'absorption par les cellules) est calculé en divisant la radioactivité restant dans les tissus par la radioactivité spécifique de la solution d'absorption.

\section{E. Déterminations quantitatives}

Après récolte, les plantes sont pesées, desséchées et pesées à nouveau. Les ions sont extraits de la matière sèche par $20 \mathrm{ml}$ de $\mathrm{HNO}_{3} 0,5$ p. 100 (72 h à température ambiante, avec agitation 4 à 5 fois par jour). Les cations sont dosés par photométrie de flamme, le chlorure par coulométrie. La radioactivité a été déterminée sur des cendres $\left(15 \mathrm{~h}\right.$ à $400{ }^{\circ} \mathrm{C}$ ) reprises dans $1 \mathrm{ml}$ de $\mathrm{HCl} 1,5 \mathrm{p}$. 100 plus $0,5 \mathrm{ml}$ d'eau, et séchées. Les mesures ont été faites à l'aide d'un compteur à circulation d'argon-méthane.

\section{RÉSULTATS}

Après $20 \mathrm{j}$ de culture sur S3 (SLAMA, 1986), les feuilles représentent chez toutes les plantes de 40 p. 100 à 60 p. 100 de la matière sèche totale, et contiennent 30 p. 100 à 60 p. $100 \mathrm{du} \mathrm{Cl}^{-}$de la plante (tabl. 2). La répartition de $\mathrm{Na}^{+}$est plus hétérogène ; les plantes sensibles sont nettement " exclusives 》 de $\mathrm{Na}^{+}$(3 p. 100 à 18 p. $100 \mathrm{du} \mathrm{Na}^{+}$total sont dans les feuilles), tandis que les plantes tolérantes sont « inclusives » (environ 30 p. 100 à 60 p. $100 \mathrm{du} \mathrm{Na}^{+}$de la plante dans les feuilles).

L'expérience suivante a pour but de comparer l'absorption de $\mathrm{Na}^{+}$et $\mathrm{Cl}^{-}$par les feuilles détachées (absorbant par l'intermédiaire de leur pétiole), et par les feuilles en place. Des feuilles sont détachées de plantes cultivées depuis $6 \mathrm{j}$ sur milieu $\mathrm{S}$, avec leur pétiole et un fragment de tige. Ce dernier est alors plongé dans le milieu S3 (le pétiole et la feuille restant à l'air). Parallèlement, des plantes cultivées depuis $6 \mathrm{j}$ sur milieu $\mathrm{S}$ sont passées sur $\mathrm{S} 3$ de manière à ce que leurs feuilles absorbent $\mathrm{Na}^{+}$et $\mathrm{Cl}^{-}$par l'intermédiaire de la racine. Après $24 \mathrm{~h}$, les masses de matières fraîche et sèche des feuilles sont mesurées, ainsi que les contenus ioniques. On n'observe aucune variation significative des teneurs en eau, ni des teneurs en $\mathrm{K}^{+}$ et $\mathrm{Ca}^{2+}$ au cours des $24 \mathrm{~h}$. Les teneurs en $\mathrm{Na}^{+}$et $\mathrm{Cl}^{-}$ des feuilles détachées ou en place sont représentées sur la figure 1. Les teneurs mesurées sur des feuilles de plantes cultivées depuis $5 \mathrm{j}$ et $10 \mathrm{j}$ sur S3 servent de points de comparaison. Les quantités d'ions accumulées en $24 \mathrm{~h}$ par les feuilles détachées sont plus importantes que celles que les feuilles en place ont accumulé pendant le même temps. Ceci montre que le transport de $\mathrm{Na}^{+}$et $\mathrm{Cl}^{-}$dans les feuilles est limité par l'absorption racinaire, la conduction longitudinale, ou les prélèvements par les autres organes. Un autre point important apparaît en comparant les quantités de $\mathrm{Na}^{+}$ accumulées en $24 \mathrm{~h}$ par les feuilles détachées aux quantités présentes après $5 \mathrm{j}$ ou $10 \mathrm{j}$ de culture sur milieu $\mathrm{S} 3$. Chez les plantes les plus résistantes à $\mathrm{NaCl}$ (Ga, Mc, Mq), les deux quantités sont du même ordre de grandeur. Au contraire, chez les plantes sensibles, les feuilles détachées se chargent en $24 \mathrm{~h}$ beaucoup plus que les feuilles en place en plusieurs jours. Ceci suggère que le faible niveau d'accumulation de $\mathrm{Na}^{+}$ dans les feuilles des plantes sensibles cultivées en présence de $\mathrm{NaCl}$ ne traduit pas uniquement la faible capacité potentielle d'accumulation de $\mathrm{Na}^{+}$. Ces plantes disposent de moyens d'empêcher le niveau d'accumulation de $\mathrm{Na}^{+}$d'atteindre sa valeur maximale dans les feuilles. Enfin, les comportements de $\mathrm{Cl}^{-}$sont très différents de ceux de $\mathrm{Na}^{+}$. L'exclusion des feuilles, typique du comportement de $\mathrm{Na}^{+}$chez les plantes sensibles, ne s'observe pas pour l'anion. Les quantités de $\mathrm{Cl}^{-}$accumulées en $24 \mathrm{~h}$ par les feuilles détachées sont toujours du même ordre de grandeur, ou plus petites, que les quantités accumulées par les feuilles en place en plusieurs jours de culture sur S3.

\section{TABLEAU 2}

Accumulations de $\mathrm{Na}^{+}$et $\mathrm{Cl}^{-}$dans les feuilles. Les plantes ont été cultivées pendant $20 \mathrm{j}$ sur milieu enrichi en $\mathrm{NaCl}$ (3 g. $\mathrm{l}^{-} \mathrm{l}$, soit $5 \mathrm{l}, 3 \mathrm{mM}$ ). Les valeurs intéressent la totalité des feuilles, sans les pétioles (moyennes de 5 plantes, traitées individuellement). Les intervalles de sécurité sont calculés au seuil $95 \%$

Accumulation of $\mathrm{Na}^{+}$and $\mathrm{Cl}^{-}$in the leaves. The plants were grown for $20 \mathrm{~d}$ in the presence of $51.3 \mathrm{mM} \mathrm{NaCl}(3 \mathrm{~g} .1$ '). Results for all leaves of each plant, without petiole (means of 5 plants, and standard error for $p=95 \%$ ).

\begin{tabular}{|c|c|c|c|c|c|c|c|c|c|c|c|c|}
\hline \multirow{3}{*}{ Traitements } & \multicolumn{12}{|c|}{ Valeurs obtenues avec les plantes suivantes } \\
\hline & & \multicolumn{2}{|c|}{ Cotonniers } & \multicolumn{3}{|c|}{ Melons } & \multicolumn{2}{|c|}{ Tournesols } & \multicolumn{2}{|c|}{ Haricots } & \multicolumn{2}{|c|}{ Courges } \\
\hline & & $\mathrm{Ga}$ & G5 & $\mathrm{Mc}$ & & $\mathrm{Mq}$ & $\operatorname{Tr}$ & $\mathrm{Ti}$ & $\mathrm{Hl}$ & $\mathrm{Hg}$ & $\mathrm{Cb}$ & $\mathrm{Cl}$ \\
\hline$\% \mathrm{MS}$ & (a) & 54 & 57 & 50 & & 47 & 45 & 43 & 50 & 56 & 56 & 54 \\
\hline$\% \mathrm{Na}^{+}$ & (b) & 57 & 29 & 27 & & 27 & 8 & 9 & 3 & 3 & 18 & 4 \\
\hline $0 \% \mathrm{Cl}^{-}$ & $(\mathrm{b})$ & 49 & 45 & 38 & & 31 & 40 & 37 & 60 & 47 & 49 & 38 \\
\hline Teneur $\mathrm{Na}^{+}$ & (c) & $714 \pm 141$ & $364 \pm 55$ & $752 \pm 158$ & 932 & \pm 188 & $242 \pm 68$ & $190)+120$ & $9 \pm 2$ & $10 \pm 7$ & $156 \pm 60$ & $30 \pm 22$ \\
\hline Teneur en $\mathrm{Cl}^{-}$ & (c) 1 & $1266 \pm 110$ & $1486 \pm 103$ & $1166 \pm 272$ & 860 & \pm 80 & $1406 \pm 183$ & $1194 \pm 140$ & $928 \pm 38$ & $702 \pm 38$ & $1718 \pm 117$ & $1142 \pm 89$ \\
\hline
\end{tabular}

(a) Matière sèche des feuilles en $\%$ de la matière sèche de la plante.

(b) Quantité d'ion dans les feuilles en $\%$ de la quantité dans la plante.

(c) Teneur dans les feuilles, en mol.g- $\mathrm{MS}$. 

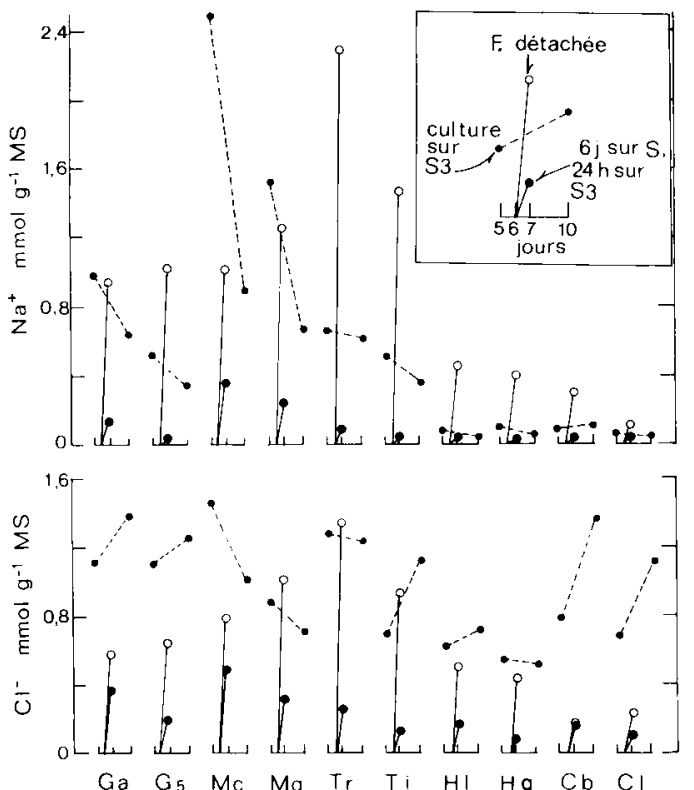

Figure 1

Accumulation de $\mathrm{Na}^{+}$et $\mathrm{Cl}^{-}$dans les feuilles. Les plantes sont cultivées $6 j$ sur solution $S$ dépourvue de $\mathrm{Na}^{+}$. Les feuilles absorbent ensuite le cation pendant $24 \mathrm{~h}$, à partir de la solution S3 (solution S plus $\left.\mathrm{NaCl} 3 \mathrm{~g} . \mathrm{l}^{-1}\right)$, soit en place sur la plante, soit détachées (absorption à travers le pétiole). Les lignes en tirets relient les teneurs mesurées dans des feuilles de plantes cultivées depuis $5 j$ et $10 \mathrm{j}$ sur S3. Les plantes (sigles donnés dans le tabl. I) sont classées de gauche à droite dans l'ordre approximatif de sensibilité croissante à $\mathrm{NaCl}$ : cotonniers $(G)$, melons $(M)$, tournesols $(T)$, haricots (H) et courges (C).

Accumulation of $\mathrm{Na}^{+}$in leaves. The plants were grown for $6 \mathrm{~d}$ on solution $S$ without $\mathrm{Na}^{+}$. Then, the leaves were allowed to take up $\mathrm{Na}^{+}$for $24 \mathrm{~h}$ from solution $\mathrm{S3}$ (solution $\mathrm{S}$ plus $3 \mathrm{g.l^{-1 }} \mathrm{NaCl}$, either on the intact plants, or detached labsorption through the petiole). The points linked by the dashed lines correspond to plants cultivated for $5 \mathrm{~d}$ or $10 \mathrm{~d}$ on S3. The two-character abbreviations of the plant names are given in table 1. From the left to the right, the plants are approximately arranged following the order of increasing sensitivity to $\mathrm{NaCl}$ : cotton $(G)$, melon $(M)$, sunflower $(T)$, bean (H) and pumpkin and marrow (C).

La vitesse d'absorption de $\mathrm{Na}^{+}$par les cellules de feuilles est mesurée chez le haricot et le cotonnier, pris respectivement comme exemples de plantes sensible et tolérante. La figure 2 montre l'incorporation du traceur ${ }^{24} \mathrm{Na}^{+}$dans les tranches de limbes de haricot et de cotonnier. Pour toutes les concentrations de $\mathrm{Na}^{+}$ étudiées, les tissus foliaires du haricot absorbent le traceur plus rapidement que ceux du cotonnier. Etant donné qu'un échange d'ions dans $\mathrm{CaSO}_{4}$ après l'incorporation du traceur a éliminé la radioactivité extracellulaire, on peut en conclure que l'influx de $\mathrm{Na}^{+}$dans les cellules du mésophylle est paradoxalement plus rapide chez l'espèce exclusive que chez l'espèce inclusive (SLAMA, 1975).

Dans une autre expérience, les feuilles sont prélevées sur des plantes cultivées depuis $6 \mathrm{j}$ sur milieu $\mathrm{S}$, et leur pétiole est plongé pendant $24 \mathrm{~h}$ dans le milieu S3. La répartition des ions entre les nervures et le limbe est ensuite examinée en disséquant les feuilles. La nervure principale et les veines secondaires saillantes sont alors découpées, donnant une fraction enrichie en tissus vasculaires; le reste, qui comprend le limbe et les veines mineures, constitue une fraction enrichie en mésophylle. Les limbes et les nervures pré-

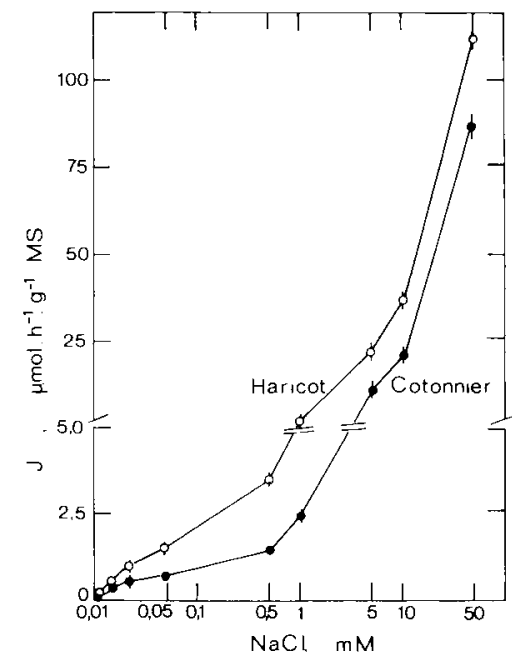

Figure 2

Influx net de sodium dans les tranches de limbes foliaires. $L$ 'absorption a lieu à la lumière et à $24^{\circ} \mathrm{C}$, à partir de solutions de $\left({ }^{24} \mathrm{Na}^{+}\right)-\mathrm{NaCl}, \mathrm{CaSO}_{4} 0,5 \mathrm{mM}$. Après $1 \mathrm{~h}$, les échantillons sont lavés pendant $30 \mathrm{~min}$ à $2{ }^{\circ} \mathrm{C}$ dans $\mathrm{NaCl} \mathrm{I} \mathrm{mM}, \mathrm{CaSO}_{4} 0,5 \mathrm{mM}$. L.'influx net est calculé à partir de la quantité de traceur restant dans les tissus.

Net influx of sodium into lamina slices. The slices absorbed ${ }^{24} \mathrm{Na}^{+}$ for $1 \mathrm{~h}$ in the light at $24^{\circ} \mathrm{C}$, from solutions containing $\mathrm{NaCl}$ and $0.5 \mathrm{mM} \mathrm{CaSO}_{4}$. They were then washed for $30 \mathrm{~min}$ at $2{ }^{\circ} \mathrm{C}$ in I $\mathrm{mM} \mathrm{NaCl}, 0.5 \mathrm{mM} \mathrm{CaSO}_{4}$ (without tracer). The net influx was calculated from the amounts of radioactivity remaining in the tissues.

sentent des teneurs en $\mathbf{K}^{+}$voisines, mais leurs teneurs en $\mathrm{Na}^{+}$diffèrent (tabl. 3). Chez les plantes résistantes, $\mathrm{Na}^{+}$est préférentiellement accumulé dans le limbe. Chez les plantes sensibles, il reste préférentiellement associé aux nervures.

\section{DISCUSSION}

Puisque les résultats obtenus avec $\mathrm{Na}^{+}$et $\mathrm{Cl}^{-}$diffèrent profondément dans les diverses expériences d'accumulation, il est clair que les feuilles accumulent les deux ions indépendamment. Seule l'accumulation de $\mathrm{Na}^{+}$présente des caractéristiques associées au degré de sensibilité des plantes à $\mathrm{NaCl}$. Le problème est de déterminer dans quelle mesure l'exclusion de $\mathrm{Na}^{+}$caractéristique des feuilles des plantes sensibles a pour cause des propriétés intrinsèques de ces organes. Les excès d'accumulation dans les feuilles détachées, par rapport aux feuilles en place, montrent que la charge ionique de ces organes est limitée par la racine et les systèmes conducteurs. L'exclusion $\mathrm{de} \mathrm{Na}^{+}$se distingue encore nettement chez les feuilles détachées alimentées à travers leur pétiole (fig. 1), ce qui indique que la racine n'est pas seule responsable de ce phénomène (SlaMA, 1986). L'exclusion de $\mathrm{Na}^{+}$est manifestement atténuée dans les feuilles détachées, car elles sont capables d'accumuler en $24 \mathrm{~h}$ plus de $\mathrm{Na}^{+}$qu'en $5 \mathrm{j}$ ou $10 \mathrm{j}$ sur la plante. Enfin, l'exclusion disparaît totalement lorsque les mésophylles sont alimentés directement à partir du milieu (fig. 2). D'après les cinétiques d'influx de ${ }^{24} \mathrm{Na}^{+}$, on peut conclure que 
TABLEAU 3

Distribution de $\mathrm{Na}^{+}$et $\mathrm{K}^{+}$entre les limbes et nervures des feuilles. Les feuilles sont prélevées avec leurs pétioles sur des plantes cultivées sur milieu sans $\mathrm{NaCl}$. Les pétioles sont plongés pendant $24 \mathrm{~h}$ dans le milieu de culture enrichi en $\mathrm{NaCl}\left(3 \mathrm{~g} . \mathrm{l}^{-1}\right.$, soit $5 \mathrm{l}, 3 \mathrm{mM}$ ). Les feuilles sont ensuite disséquées en " nervures" (veines principales) et "limbes " (mésophylles et veines mineures). Les valeurs sont les moyennes de 5 répétitions (intervalles de sécurité au seuil de $95 \%$ ).

Distribution of $\mathrm{Na}^{+}$and $\mathrm{K}^{+}$in the lamina and veins of leaves. The leaves were excised with their petioles from plants grown on medium $\mathrm{S}$ (without $\mathrm{NaCl}$ ). The cut extremities of the petioles were immersed for $24 \mathrm{~h}$ in the culture solution complemented with $51.3 \mathrm{mM}$ NaCl (3 g. ${ }^{-1}$ ). The "veins" (main veins) and "lamina" (mesophylls and minor veins). were then dissected. Means of 5 replicates and standard errors for $p=95 \%$

\begin{tabular}{|c|c|c|c|c|c|c|}
\hline \multirow{3}{*}{$\begin{array}{c}\text { Ions et } \\
\text { tissus }\end{array}$} & \multicolumn{6}{|c|}{ Teneurs obtenues avec les plantes suivantes ( $\mu \mathrm{mol} . \mathrm{g}^{-1} \mathrm{MS}$ ) } \\
\hline & \multicolumn{2}{|c|}{ Cotonniers } & \multicolumn{2}{|c|}{ Haricots } & \multicolumn{2}{|c|}{ Courges } \\
\hline & Ga & G5 & $\mathrm{Hl}$ & $\mathrm{Hg}$ & $\mathrm{Cb}$ & $\mathrm{Cl}$ \\
\hline $\mathbf{K}^{+}$ & $1150 \pm 40$ & $910 \pm 11$ & $1050 \pm$ & $1460 \pm 110$ & $1060 \pm 40$ & $1050 \pm 170$ \\
\hline $\mathrm{K}^{+}$nervures & $1220 \pm 110$ & $830 \pm 160$ & $1410 \pm 90$ & $1480 \pm 170$ & $1150 \pm 150$ & $1310 \pm 150$ \\
\hline $\mathrm{Na}^{+}$nervures & $850 \pm 90$ & $750 \pm 180$ & $1020 \pm 70$ & $930 \pm 90$ & $400 \pm 130$ & $340 \pm 150$ \\
\hline
\end{tabular}

pour une même concentration dans l'apoplasme, le mésophylle de la plante sensible absorbe $\mathrm{Na}^{+}$plus vite que celui de la plante tolérante. Pour expliquer la faible accumulation chez la plante sensible, il faut donc supposer que la concentration de $\mathrm{Na}^{+}$dans l'apoplasme de ses feuilles est maintenu plus basse que chez la plante tolérante, et que ceci ne résulte pas uniquement d'une moindre alimentation par la racine (puisque l'exclusion se distingue chez les feuilles détachées). L'hypothèse la plus plausible est que le $\mathrm{Na}^{+}$apporté par le xylème n'atteint pas le mésophylle parce qu'il est fortement retenu dans les tissus vasculaires. Les données du tableau 3 appuient cette hypothèse, qui est en accord également avec les résultats de JАCOBY (1975). L'efficacité particulière du phloème des plantes sensibles à absorber et transporter $\mathrm{Na}^{+} \mathrm{a}$ effectivement été démontrée à l'aide de plusieurs techniques (WALLACE, 1963 ; COOIL et al., 1965 ; LEVI, 1970 ; LESSANI \& MARSCHNER, 1978 ; WINTER, $1982 a$ et $b$; Winter \& Preston, 1982).

En résumé, le caractère exclusif vis-à-vis de $\mathrm{Na}^{+}$ caractéristique des plantes sensibles à $\mathrm{NaCl}$, se mani- feste pleinement dans les organes en place, il est atténué chez les feuilles détachées absorbant à travers leur pétiole, et disparaît lorsque les limbes absorbent directement à partir du milieu. L'ensemble de ces observations, rapproché des résultats sur la répartition de $\mathrm{Na}^{+}$entre limbes et nervures, et des données de la littérature sur le transport descendant de $\mathrm{Na}^{+}$, supporte l'hypothèse que les caractères inclusif et exclusif sont sous la dépendance des systèmes de conduction (GREenwaY \& MunNS, 1980). Cette hypothèse prévoit que l'aptitude à remettre en circulation dans le phloème le $\mathrm{Na}^{+}$qui atteint la feuille est en relation positive avec la sensibilité des plantes à $\mathrm{NaCl}$.

\section{REMERCIEMENTS}

Je remercie Monsieur le Professeur C. Grignon (ENSA-INRA, Montpellier), pour son aide dans l'interprétation des résultats.

Reçu le 2 février 1986. Accepté le 31 mars 1987.

\section{RÉFÉRENCES BIBLIOGRAPHIQUES}

Bernstein L., 1964. Effect of salinity on mineral composition and growth of plants. Plant Anal. Fertil. Probl., 4, 25-45.

Collander R., 1941. Selective absorption of cations by higher plants. Plant Physiol., 16, 691-720.

Cooil B. J., De La Fuente R. K., De La Pena R. S., 1965. Absorption and transport of sodium and potassium in the squash. Plant Physiol., 40, 625-632.

Greenway H., Munns R., 1980. Mechanisms of salt tolerance in nonhalophytes. Ann. Rev. Plant Physiol., 31, 149-190.

Hamza M., 1977. Action de différents régimes d'apport de chlorure de sodium sur la physiologie de deux légumineuses: Phaseolus vulgaris (sensible) et Hedysarum carnosum (tolérante). Relations hydriques et relations ioniques. Thèse de Doctorat d'Etat, Université de Paris VIl, pp. 252.

Jacobson L., 1951. Maintainance of iron supply in nutrient solutions by a single addition of ferric potassium ethylenediaminetetraacetate. Plant Physiol., 26, 411-413.

Jacoby B., 1975. Light sensitivity of ${ }^{22} \mathrm{Na},{ }^{86} \mathrm{Rb}$ and ${ }^{42} \mathrm{~K}$ absorption by different tissues of bean leaves. Plant Physiol., 55, 978-981.
Lessani H., Marschner H., 1978. Relation between salt tolerance and long-distance transport of sodium and chloride in various crop species. Aust. J. Plant Physiol., 5, 27-37.

Levi E., 1970. Distribution du sodium absorbé simultanément par voies racinaire et foliaire dans une jeune plante de haricot (Phaseolus vulgaris var. Berna) manifestée par la répartition de deux de ses isotopes radioactifs. C.R. Acad. Sci., 270, Série D, 2798-2801.

Pearson G. A., 1964. Effects of inhibition on sodium absorption and translocation in bean and cotton. Plant Physiol., S39, 12

Slama F., 1974. Absorption du sodium et du potassium par des fragments de limbe et de tige du haricot (Phaseolus vulgaris L.) et $d u$ cotonnier (Gossypium hirsutum L.). Thèse $3^{\text {e }}$ Cycle, Université de Paris VII, pp. 99.

Slama F., 1975. Absorption et exsorption du sodium par des fragments de limbe et de tige du haricot et du cotonnier. C.R. Acad. Sci., 280, Série D, 37-40.

Slama F., 1982. Effets de $\mathrm{NaCl}$ sur la croissance et la nutrition minérale de six espèces de plantes cultivées. Thèse de Doctorat d'Etat, Tunis, pp. 214. 
Slama F., 1986. Intervention des racines dans la sensibilité ou la tolérance à NaCl de plantes cultivées. Agronomie, 6 (7), 651-658.

Winter E., 1982a. Salt tolerance of Trifolium alexandrinum L. II. Ion balance in relation to its salt tolerance, Aust. J. Plant Physiol,, 9, 227-237.

Winter E., 1982 $b$. Salt tolerance of Trifolium alexandrinum L. III. Effects of salt on ultrastructure of phloem and xylem transfer cells in petioles and leaves. Aust. J. Plant Physiol., 9, 239-250.
Winter E., Preston J., 1982. Salt tolerance of Trifolium alexandrinum L. IV. Ion measurements by X-ray microanalysis in unfixed, frozen hydrated leaf cells at various stages of salt treatment. Aust. J. Plant Physiol., 9, 251-259.

Yeo A. R., 1983. Salinity resistance: physiology and prices. Physiol. Plant., 58, 214-222. 